\title{
Automatic Wood Species Identification of Korean Softwood Based on Convolutional Neural Networks ${ }^{1}$
}

\author{
Ohkyung Kwon $\mathbb{D}^{2, \dagger} \cdot$ Hyung Gu Lee ${ }^{2} \cdot$ Mi-Rim Lee ${ }^{2} \cdot$ Sujin Jang ${ }^{2} \cdot$ \\ Sang-Yun Yang $^{3} \cdot$ Se-Yeong Park ${ }^{3} \cdot$ In-Gyu Choi ${ }^{3,4,5} \cdot$ Hwanmyeong Yeo ${ }^{3,4}$
}

\begin{abstract}
Automatic wood species identification systems have enabled fast and accurate identification of wood species outside of specialized laboratories with well-trained experts on wood species identification. Conventional automatic wood species identification systems consist of two major parts: a feature extractor and a classifier. Feature extractors require hand-engineering to obtain optimal features to quantify the content of an image. A Convolutional Neural Network $(\mathrm{CNN})$, which is one of the Deep Learning methods, trained for wood species can extract intrinsic feature representations and classify them correctly. It usually outperforms classifiers built on top of extracted features with a hand-tuning process.

We developed an automatic wood species identification system utilizing CNN models such as LeNet, MiniVGGNet, and their variants. A smartphone camera was used for obtaining macroscopic images of rough sawn surfaces from cross sections of woods. Five Korean softwood species (cedar, cypress, Korean pine, Korean red pine, and larch) were under classification by the CNN models. The highest and most stable CNN model was LeNet3 that is two additional layers added to the original LeNet architecture. The accuracy of species identification by LeNet3 architecture for the five Korean softwood species was $99.3 \%$. The result showed the automatic wood species identification system is sufficiently fast and accurate as well as small to be deployed to a mobile device such as a smartphone.
\end{abstract}

Keywords : automatic wood species identification, convolutional neural networks, CNN, LeNet, MiniVGGNet

${ }^{1}$ Date Received October 5, 2017, Date Accepted October 30, 2017

2 National Instrumentation Center for Environmental Management (NICEM), Seoul National University, 1 Gwanak-ro, Gwanak-gu, Seoul 08826, Republic of Korea

3 Department of Forest Sciences, Seoul National University, 1 Gwanak-ro, Gwanak-gu, Seoul 08826, Republic of Korea

${ }^{4}$ Research Institute of Agriculture and Life Sciences, Seoul National University, 1 Gwanak-ro, Gwanak-gu, Seoul 08826, Republic of Korea

5 Institutes of Green Bio Science and Technology, Seoul National University, 1447 Pyeongchang-daero, Daehwa-myeon, Pyeongchang 25354, Republic of Korea

${ }^{\dagger}$ Corresponding author: Ohkyung Kwon (e-mail: zoom@snu.ac.kr, ORCID: 0000-0002-6307-0060) 
Ohkyung Kwon $\cdot$ Hyung Gu Lee $\cdot$ Mi-Rim Lee $\cdot$ Sujin Jang $\cdot$ Sang-Yun Yang $\cdot$ Se-Yeong Park $\cdot$ In-Gyu Choi $\cdot$ Hwanmyeong Yeo

\section{INTRODUCTION}

Automatic wood species identification systems have enabled fast and accurate identification of wood species outside of specialized laboratories with well-trained experts on wood species identification. Previously most of the automated wood species identification systems have used pipelines relying on hand-tuning segmentation, feature extraction and classification steps for each macroscopic images of the wood surface.

Feature extractors or image descriptors require hand-engineering to obtain optimal features to quantify the content of an image. In general, image content quantification algorithms can be classified into several categories such as encoding color (color moments, color histograms, color correlograms), encoding shape (Hu moments and Zernike moments), encoding texture (Local Binary Pattern and Haralick texture), key point detectors (FAST, Harris, DoG, and so on), local invariant descriptors (SIFT, SURF, BRIEF, ORB, and so on), and Histogram of Oriented Gradients (HOG).

For the past years, many researchers have explored various types of feature extractors for wood identification: hue, saturation, value, contrast, angular second moment, sum of variances, long run emphasis, fractal dimension, and wavelet horizontal energy proportion ( $\mathrm{Yu}$ et al., 2009), color-based features (Peng, 2013), texture-based features such as Gabor filters, Gray Level Co-occurrence Matrices (GLCM), Local Binary Patterns (LBP), Completed Local Binary
Pattern (CLBP), Local Phase Quantization (LPQ), Basic Grey Level Aura Matrix (BGLAM), Improved Basic Grey Level Aura Matrix (I-BGLAM), Statistical Properties of Pores Distribution (SPPD), Mask Matching Image (MMI), Coiflet Discrete Wavelet Transform (DWT), the Markovian, spectral, and illumination invariant textural features, anisotropic diffusion and Local Directional Binary Patterns (LDBP) (Tou et al., 2009a; Tou et al., 2009b; Nasirzadeh et al., 2010; Yusof et al., 2010; Yusof and Rosli, 2013; Kobayashi et al., 2015; Kobayashi et al., 2017; Khalid et al., 2011; Khairuddin et al., 2011, Wang et al., 2013a; Wang et al., 2013b; Yadav et al., 2013; Mohan et al., 2014; Yadav et al., 2014; Martins et al., 2015; Haindl and Vácha, 2015; Zamri et al., 2016; Hiremath and Bhusnurmath, 2017), key point detectors, and local invariant descriptors such as Speeded Up Robust Features (SURF) (Huang et al., 2009) and Scale-Invariant Feature Transform (SIFT) (Hu et al., 2015; Martins et al., 2015). A Kernel-Genetic Algorithm (K-GA) technique was also used for feature selection (Yusof et al., 2013a).

From the extracted feature vectors, researchers used different types of classifiers such as $\mathrm{k}$ Nearest-Neighbor $(\mathrm{kNN})$ classifier (Khalid et al., 2011; Khairuddin et al., 2011; Kobayashi et al., 2015; Hu et al., 2015; Hiremath and Bhusnurmath, 2017), Support Vector Machine (SVM) (Martins et al., 2013; Paula Filho et al., 2014; Hu et al., 2015; Zamri et al., 2016), Linear Discriminant Analysis (LDA) classifier (Khalid et al., 2011), a multi-layer neural net- 
work based on the popular back propagation (MLBP) algorithm (Yusof et al., 2010; Yusof and Rosli, 2013), Artificial Neural Networks (ANN) (Hu et al., 2015), Multilayer Perceptron Backpropagation Artificial Neural Network (MPB ANN) (Yadav et al., 2013), several WEKA classification algorithms (Yadav et al., 2014), and correlation (Mohan et al., 2014). Sometimes, a pre-classifier such as Fuzzy logic-based pre-classifier (Yusof et al., 2013b) was used to increase classification accuracy. For the same purpose, several studies adapted combinatory strategies such as image segmentation and multiple feature sets (Cavalin et al., 2013; Kapp et al., 2014), a two-level divide-and-conquer classification strategy (Paula Filho et al., 2014), the combination of all classifiers, and different dynamic selection of classifiers (DSC) methods (Martins et al., 2015). Also, an adaptive multi-level approach for combining multiple classifications was applied to forest species recognition (Cavalin et al., 2016).

In these days, the hand-engineering process in the conventional automatic image recognition has been replaced by utilizing Convolutional Neural Networks (CNNs) such as LeNet (Lecun et al., 1998), AlexNet (Krizhevsky et al., 2012), GoogLeNet (Szegedy et al., 2014), VGGNet (Simonyan and Zisserman, 2014), ResNet (He et al., 2016), and so on. The CNNs trained for wood species can extract intrinsic feature representations and classify them correctly. It usually outperforms classifiers built on top of extracted features with a hand-tuning process. A CNN model was developed to rec- ognize Brazilian forest species (macroscopic images for 41 species and microscopic images for 112 species), and resulting accuracy was better than $97 \%$ for both datasets (Hafemann et al., 2014).

Utilization of a mobile device like a smartphone is essential to speed up the wood identification process on sites. A smartphone equips with a decent camera can be used as a camera for automatic wood species identification from macroscopic pictures of wood although it is not the best choice for the conventional process of feature extraction tasks. Also, illumination condition is often not appropriate for most of the traditional image recognition tasks. However, deep neural network techniques give a chance to overcome the limitations posed by the conventional feature extraction methods requiring high-quality images under controlled illumination.

In this study, we developed an automatic wood species identification system utilizing CNN models and macroscopic images that were obtained by a smartphone camera. Regarding the accuracy of the automatic wood species identification system, several pipelines based on different $\mathrm{CNN}$ models were evaluated for five Korean wood species (cedar, cypress, Korean pine, Korean red pine, and larch).

\section{MATERIALS and METHODS}

\subsection{Sample Preparation}

Five Korean softwood species [cedar (Cryptomeria japonica), cypress (Chamaecyparis 
Table 1. A number of images $(512 \times 512$ pixels $)$ for training and testing purpose for each species

\begin{tabular}{cccc}
\hline Species & Train & Test & Total \\
\hline \hline cedar & 2321 & 774 & 3095 \\
\hline cypress & 2565 & 855 & 3420 \\
\hline Korean pine & 2970 & 990 & 3960 \\
\hline Korean red pine & 2295 & 765 & 3060 \\
\hline Larch & 2498 & 832 & 3330 \\
\hline Total & 12649 & 4216 & 16865 \\
\hline
\end{tabular}

obtusa), Korean pine (Pinus koraiensis), Korean red pine (Pinus densiflora), and larch (Larix kaempferi)] were under investigation by an automatic wood species identification utilizing $\mathrm{CNN}$ techniques. We purchased fifty lumbers of each species of $50 \times 100 \times 1200 \mathrm{~mm}^{3}$ (thickness $\times$ with $\times$ length) from several mills participating the National Forestry Cooperative Federation in Korea. The lumbers in each species were from different regions of Korea. $10 \sim 20$ specimens of $40 \times 50 \times 100 \mathrm{~mm}^{3}(\mathrm{R} \times \mathrm{T} \times \mathrm{L})$ were cut from each lumber (50 wood samples per species).

\subsection{Image Acquisition and Dataset Preparation}

We used a smartphone (iPhone 7) to obtain macroscopic pictures of the sawn surfaces of cross sections of the specimen. During image acquisition process, the smartphones placed on a simple frame as a stable support. The camera in an iPhone 7 model has f/1.8 lens and phase detection autofocus function and produces an image of 12 Megapixels. The camera produces a color picture of $3024 \times 4032$ pixels. The pixel size of the image was $41.7 \mu \mathrm{m}$. Only center

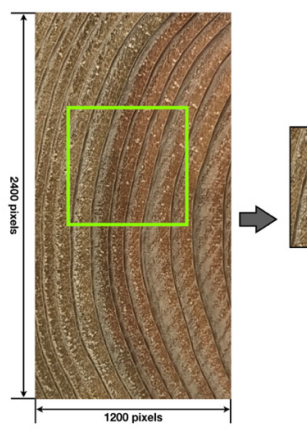

(A)

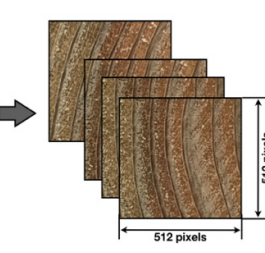

(B)

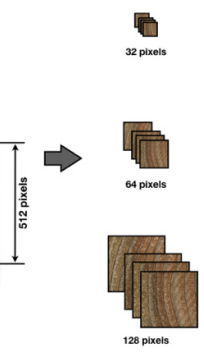

(C)
Fig. 1. Dataset preparation from five Korean softwood species. (A) Cropped image from an original image from a smartphone camera, (B) cropped images prepared from (A) by a sliding window method, and (C) resized images from (B). Images of (C) step were input images for the CNN models used in this study.

part of the picture contains an image of wood. The images were vertical shape, and thus only a part of the wood image $(1200 \times 2400$ pixels around the center) was cropped. The total number of the cropped images were 187.

We prepared 16865 images of $512 \times 512$ pixels by utilizing a sliding window method; 12649 images of all (75\%) were used for training and the other 4216 images (25\%) for validation. Table 1 listed the number of images for each species. Also, we separately prepared an "External Validation Set (EVS)" for determination of the accuracy of the automated wood species identification. The images in the EVS were not overlapped those in the training and testing sets. The EVS included total 50 images (10 images from each species) of $1200 \times 2400$ pixels. 
Table 2. A table summary of the LeNet architecture for $32 \times 32 \times 3$ input image. To build LeNet2 and LeNet3, one layer or two layers were added to the LeNet architecture, respectively

\begin{tabular}{ccc}
\hline Layer Type & Output Size & $\begin{array}{c}\text { Filter Size } \\
\text { /Stride }\end{array}$ \\
\hline \hline INPUT IMAGE & $32 \times 32 \times 3$ & \\
\hline CONV & $32 \times 32 \times 20$ & $5 \times 5 \times 20$ \\
\hline ACT & $32 \times 32 \times 20$ & \\
\hline POOL & $16 \times 16 \times 20$ & $2 \times 2$ \\
\hline CONV & $16 \times 16 \times 50$ & $5 \times 5 \times 50$ \\
\hline ACT & $16 \times 16 \times 50$ & \\
\hline POOL & $8 \times 8 \times 50$ & \\
\hline FC & 500 & \\
\hline ACT & 500 \\
\hline FC & 5 \\
\hline SOFTMAX & 5 \\
\hline
\end{tabular}

\subsection{Model Architecture}

The CNNs are the types of algorithms that can learn appropriate parameters of various image processing operations such as smoothing, sharpening, and edge detection for an input image. The CNNs also have capabilities to automatically learn discriminating filters for detection of low-level structures such as edges and blob-like structures as well as of high-level objection such as faces, cats, dogs, cups, etc. This utilization of the lower-level layers or features to learn high-level features is called the compositionality of CNNs, which is achieved by stacking a specific set of layers purposefully. Building blocks of CNNs are convolution (CONV) layer, activation (ACT) layer, pooling (POOL) layer, fully-connected (FC) layer, batch normalization $(\mathrm{BN})$, and dropout (DROPOUT). Combinations of these building
Table 3. A table summary of MiniVGGNet architectures for $32 \times 32 \times 3$ input image. To build MiniVGGNet2 and MiniVGGNet3, one layer or two layers were added to the MiniVGGNet architecture, respectively

\begin{tabular}{|c|c|c|}
\hline Layer Type & Output Size & $\begin{array}{c}\text { Filter Size } \\
/ \text { Stride }\end{array}$ \\
\hline INPUT IMAGE & $32 \times 32 \times 3$ & \\
\hline CONV & $32 \times 32 \times 32$ & $3 \times 3 \times 32$ \\
\hline ACT & $32 \times 32 \times 32$ & \\
\hline $\mathrm{BN}$ & $32 \times 32 \times 32$ & \\
\hline CONV & $32 \times 32 \times 32$ & $3 \times 3 \times 32$ \\
\hline ACT & $32 \times 32 \times 32$ & \\
\hline $\mathrm{BN}$ & $32 \times 32 \times 32$ & \\
\hline POOL & $16 \times 16 \times 32$ & $2 \times 2$ \\
\hline DROPOUT & $16 \times 16 \times 32$ & \\
\hline CONV & $16 \times 16 \times 64$ & $3 \times 3 \times 64$ \\
\hline ACT & $16 \times 16 \times 64$ & \\
\hline $\mathrm{BN}$ & $16 \times 16 \times 64$ & \\
\hline CONV & $16 \times 16 \times 64$ & $3 \times 3 \times 64$ \\
\hline ACT & $16 \times 16 \times 64$ & \\
\hline $\mathrm{BN}$ & $16 \times 16 \times 64$ & \\
\hline POOL & $8 \times 8 \times 64$ & $2 \times 2$ \\
\hline DROPOUT & $8 \times 8 \times 64$ & \\
\hline $\mathrm{FC}$ & 512 & \\
\hline $\mathrm{ACT}$ & 512 & \\
\hline $\mathrm{BN}$ & 512 & \\
\hline DROPOUT & 512 & \\
\hline $\mathrm{FC}$ & 5 & \\
\hline SOFTMAX & 5 & \\
\hline
\end{tabular}

blocks become a $\mathrm{CNN}$ architecture for a given task (Table 2 and 3).

LeNet and VGGNet were the base of the models investigated in this study. LeNet architecture is simple with only two convolution layers (Fig. 2 and Table 2). VGGNet is a deep CNN (16 or 19 layers), but we stripped VGGNet down to only with two layers of two convolutional layers to build MiniVGGNet (Fig. 2 and Table 3). We added third and fourth ex- 

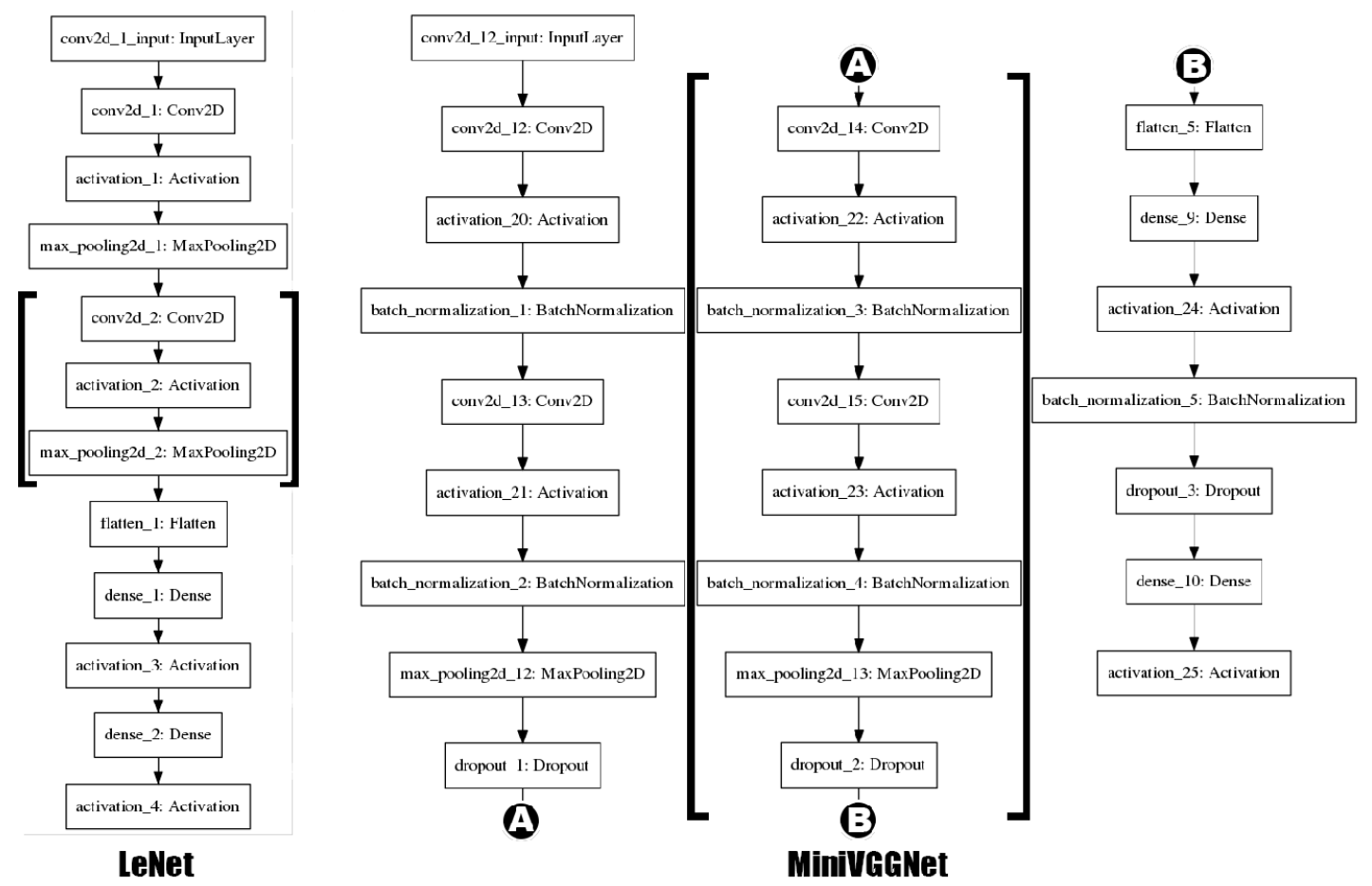

Fig. 2. The LeNet and MiniVGGNet architectures. The layers between brackets are the extensive unit layer for each architecture during extension of the model.

tensive layers into the base models to build LeNet2, LeNet3, MiniVGGNet2, and MiniVGGNet3. For the LeNet-based model, $(\mathrm{CONV} \Longrightarrow \mathrm{ACT} \Longrightarrow$ POOL) was the extensive layer unit, but for the MiniVGGNet model, $(\mathrm{CONV} \Longrightarrow \mathrm{ACT} \Longrightarrow \mathrm{BN} \Longrightarrow \mathrm{CONV} \Longrightarrow \mathrm{ACT} \Longrightarrow$ $\mathrm{BN} \Longrightarrow \mathrm{POOL} \Longrightarrow$ DROPOUT) was the one.

\subsection{Model training and determination of accuracy}

The Stochastic Gradient Descent (SGD) algorithm optimized the model parameters with the learning rate $=0.01$. The loss function was binary cross entropy. A number of epochs were 50 with a batch size of 64 . Training process used three levels $(32,64$, and 128 pixels) of input images. Pixel values of the input images were normalized by 255. A workstation with XEON CPU (28 threads) with 64 GB of memory as well as GPU with 24 GB (NVIDIA Quadro M6000). The operating system was Ubuntu 16.04 LTS with CUDA 8.0, Python 2.7, Tensorflow 1.2 and Keras 2.0.

We evaluate identification performance of the CNNs by utilizing the following equation.

Accuracy $(\%)=\frac{\begin{array}{c}\text { Number of correctly } \\ \text { identified patches }\end{array}}{\begin{array}{c}\text { Total number of patches } \\ \text { used for identification }\end{array}} \times 100$


Table 4. The accuracy (\%) of the CNN models with image size $=32$. The Accuracy Test Set was used for accuracy test for automatic identification of the five Korean softwoods

\begin{tabular}{ccccccc}
\hline Species & LeNet & LeNet2 & LeNet3 & MiniVGGNet & MiniVGGNet2 & MiniVGGNet3 \\
\hline \hline Cedar & 97.1 & 85.4 & 99.5 & 99.9 & 99.7 & 100.0 \\
\hline Cypress & 51.4 & 83.9 & 79.2 & 25.4 & 79.0 & 62.0 \\
\hline Korean Pine & 27.3 & 46.7 & 59.7 & 20.6 & 49.2 & 35.7 \\
\hline Korean Red Pine & 85.3 & 81.7 & 84.4 & 35.1 & 40.3 & 35.6 \\
\hline Larch & 99.3 & 99.7 & 98.2 & 25.0 & 38.0 & 43.2 \\
\hline Average & $\mathbf{7 2 . 1}$ & $\mathbf{7 9 . 5}$ & $\mathbf{8 4 . 2}$ & $\mathbf{4 1 . 2}$ & $\mathbf{6 1 . 2}$ & $\mathbf{5 5 . 3}$ \\
\hline Standard Deviation & 28.2 & 17.6 & 14.5 & 29.7 & 24.2 & 24.3 \\
\hline
\end{tabular}

Table 5. The accuracy (\%) of the CNN models with image size $=64$. The Accuracy Test Set was used for accuracy test for automatic identification of the five Korean softwoods

\begin{tabular}{ccccccc}
\hline Species & LeNet & LeNet2 & LeNet3 & MiniVGGNet & MiniVGGNet2 & MiniVGGNet3 \\
\hline \hline Cedar & 99.1 & 99.6 & 100.0 & 100.0 & 100.0 & 94.6 \\
\hline Cypress & 83.0 & 93.1 & 99.8 & 90.9 & 95.7 & 92.2 \\
\hline Korean Pine & 90.9 & 93.5 & 63.7 & 99.8 & 98.6 & 99.3 \\
\hline Korean Red Pine & 97.0 & 96.1 & 96.4 & 76.5 & 87.0 & 82.4 \\
\hline Larch & 92.6 & 94.8 & 98.3 & 81.6 & 78.4 & 80.3 \\
\hline Average & $\mathbf{9 2 . 5}$ & $\mathbf{9 5 . 4}$ & $\mathbf{9 1 . 6}$ & $\mathbf{8 9 . 8}$ & $\mathbf{9 1 . 9}$ & $\mathbf{8 9 . 8}$ \\
\hline Standard Deviation & 5.6 & 2.3 & 14.0 & 9.5 & 8.1 & 7.3 \\
\hline
\end{tabular}

From the EVS (images of $1200 \times 2400$ pixels), we randomly cropped 100 patches with $512 \times 512$ pixels. We chose the size of the patch to include wood anatomical features such as growth rings. Each patch was fed into the model generated by utilizing the model architecture described in section 2.3. For each prediction, we examined the classification result whether it is true or false, and then calculated an accuracy of wood species identification by the model.

To utilize macroscopic features of different wood species, we need to make the patch images to include at least several growth rings. We determined the patch size according to the given condition for macroscopic features of all wood species. In the case of the smartphone camera without a zoom factor, the field of view in $512 \times 512$ pixels was turned out to be a proper size.

\section{RESULTS and DISCUSSION}

\subsection{Accuracy of the CNN models}

In general, the accuracy of the CNN models was improved by size increase of the input image (Table 4, 5, and 6; Fig. 3). With image size $=32$, average accuracies of LeNet, LeNet2, LeNet3, MiniVGGNet, MiniVGGNet2, and 
Ohkyung Kwon $\cdot$ Hyung Gu Lee $\cdot$ Mi-Rim Lee $\cdot$ Sujin Jang $\cdot$ Sang-Yun Yang $\cdot$ Se-Yeong Park $\cdot$ In-Gyu Choi $\cdot$ Hwanmyeong Yeo

Table 6. The accuracy (\%) of the $\mathrm{CNN}$ models with image size $=128$. The Accuracy Test Set was used for accuracy test for automatic identification of the five Korean softwoods

\begin{tabular}{ccccccc}
\hline Species & LeNet & LeNet2 & LeNet3 & MiniVGGNet & MiniVGGNet2 & MiniVGGNet3 \\
\hline \hline Cedar & 94.9 & 98.7 & 100.0 & 99.1 & 99.3 & 99.9 \\
\hline Cypress & 96.5 & 97.7 & 98.2 & 99.5 & 99.7 & 100.0 \\
\hline Korean Pine & 89.3 & 93.4 & 99.0 & 91.2 & 89.9 & 90.8 \\
\hline Korean Red Pine & 95.2 & 98.7 & 99.9 & 90.1 & 99.1 & 96.7 \\
\hline Larch & 94.7 & 98.3 & 99.6 & 96.0 & 99.3 & 100.0 \\
\hline Average & $\mathbf{9 4 . 1}$ & $\mathbf{9 7 . 4}$ & $\mathbf{9 9 . 3}$ & $\mathbf{9 5 . 2}$ & $\mathbf{9 7 . 5}$ & $\mathbf{9 7 . 5}$ \\
\hline Standard Deviation & 2.5 & 2.0 & 0.7 & 3.9 & 3.8 & 3.6 \\
\hline
\end{tabular}

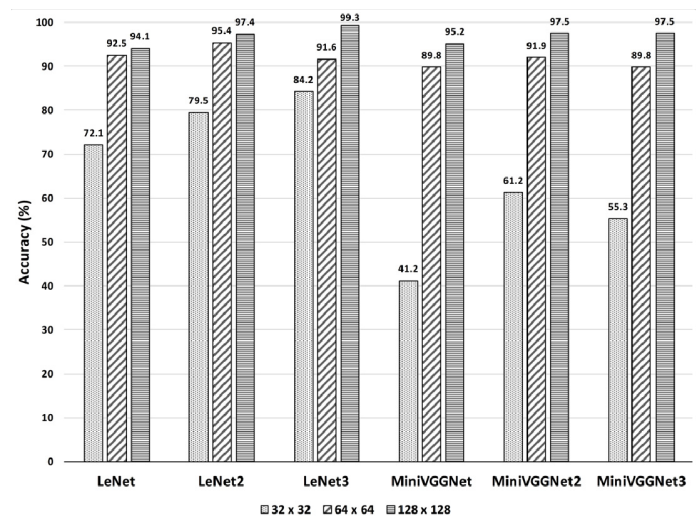

Fig. 3. Performance of $\mathrm{CNN}$ models for identification of five Korean softwood species. Utilizing bigger image size, higher accuracy is achieved. MiniVGGNet architecture is more sensitive than LeNet architecture to the changes of input image size for $\mathrm{CNN}$.

MiniVGGNet3 were $(72.1,79.5,84.2,41.2$, 61.2 , and $55.3 \%$ ), respectively. With patch size $=64$ and 128, they increased to $(92.5,95.4$, 91.6, 89.8, 91.9, and 89.8\%), and (94.1, 97.4, $99.3,95.2,97.5$, and 97.5\%), respectively. The accuracy improvement by the increase of the input image is related to the disappearance of minute details of image content during the resizing process.

With the addition of layers to the original
LeNet and MiniVGGNet, accuracy improvement was not apparent with image size $=32$ and 64 (Table 4 and 5). With image size $=128$, improvement of accuracy was evident for the LeNet-based model, but not consistent with the MiniVGGNet models (Table 6).

All MiniVGGNet-based models with patch size $=128$ showed very high average accuracy, but the standard deviation was greater than $3 \%$. The highest accuracy $(99.3 \pm 0.7 \%)$ was obtained by LeNet 3 model with image size $=128$. Thus, LeNet3 was considered to be the best $\mathrm{CNN}$ model for identification of the five Korean softwood species (Table 6).

There was no clear trend in accuracy improvement related to wood species, but the LeNet-based and MiniVGGNet-based models gave the best identification accuracy for different species. With image size $=128$, LeNet3 model showed $100 \%$ accuracy for cedar, but MiniVGGNet3 showed 100\% accuracy for cypress and Korean red pine (Table 6). While MiniVGGNet3 showed only $90.8 \%$ for Korean pine, LeNet3 produced greater than $98 \%$ for all other species. Thus, it is clear that LeNet3 was 
the best species identification CNN model for the five Korean softwoods.

\subsection{Effect of Input Images}

Improvement of identification accuracy according to the size of input images was apparent (Fig. 3). All models showed the lowest accuracy with the smallest image size $(32 \times 32$ pixels). When the images of $512 \times 512$ pixels were resized into $32 \times 32$ pixels image, most of the details of the images considered to be disappeared. No distinctive features from the small images were not learned to differentiate the wood species. By the increase of the size of the input image, more features remained. As a result, the identification accuracy was improved. With this fashion, bigger size of input images might improve accuracy more, but computational cost increases. If we collect more images and expand the number of classes for identification, input images might not be loaded into the computer memory. We need to consider computational cost, a number of classes, and a number of images in each class whether the image size needs to be increased further or not. At the moment, the LeNet 3 model produced $>$ $98 \%$ accuracy for all five species; image size = 128 was considered to be sufficient for the use of this automatic wood species identification with a macroscopic image from a camera in an iPhone 7 smartphone.

Quality of a smartphone camera is a major factor to affect the accuracy of the automatic wood species identification. The image qualities of different smartphone cameras were not investigated in this study, but any smartphone with a decent camera module is expected to obtain images with sufficient quality of macroscopic images from a rough sawn surface of the wood. The illumination condition used in this study was controlled to produce not much of shade, but the image acquisition setup utilized in this study was straightforward to make an auxiliary attachment for a smartphone. With a proper auxiliary attachment for a given smartphone, we can quickly reproduce the quality of macroscopic images.

\section{CONCLUSION}

In this study, we investigated the use of deep learning techniques to automatically identify wood species of five Korean softwoods (cedar, cypress, Korean pine, Korean red pine, and larch). We built six CNN models (LeNet, LeNet2, LeNet3, MiniVGGNet, MiniVGGNet2, and MiniVGGNet3) and trained the models for the five species. A smartphone camera was used to obtain macroscopic images from rough sawn surfaces of the cross sections of the softwoods.

The experimental results showed that the best accuracy $(99.3 \%)$ was achieved by LeNet3 model trained with macroscopic images captured by iPhone 7 camera. The MiniVGGNet3 model produced $97.5 \%$ accuracy on the same dataset of the softwoods, but the standard deviation was much larger $(3.6 \%)$ than that of LeNet3 model (0.7\%). 
Ohkyung Kwon $\cdot$ Hyung Gu Lee $\cdot$ Mi-Rim Lee $\cdot$ Sujin Jang $\cdot$ Sang-Yun Yang $\cdot$ Se-Yeong Park $\cdot$ In-Gyu Choi $\cdot$ Hwanmyeong Yeo

We demonstrated higher than 99\% accuracy of wood species identification with a deep $\mathrm{CNN}$ model with four convolutional layers. The result in this study proved that a fast and accurate automatic wood species identification system could be developed by utilizing deep CNN models. The weights produced by the $\mathrm{CNN}$ models were small enough to be installed on a mobile device such as a smartphone. Deploying a mobile device with an automatic wood species identification capability can relieve the issues of slow process to obtain an accurate wood species identification due to lack of well-trained field agents, which causes the delay of logistics.

\section{ACKNOWLEDGEMENTS}

The Forest Science \& Technology Projects (Project No. 2016009D10-1719-AB01) provided by the Korea Forest Service financially supported this study.

\section{REFERENCES}

Cavalin, P.R., Kapp, M.N., Oliveira, L.E.S. 2016. An adaptive multi-level framework for forest species recognition. Proc. of 2015 Brazillian Conference on Intelligent Systems (BRAICS 2015), pp. 92 $\sim 97$.

Cavalin, P.R., Martins, J., Kapp, M.N., Oliveira, L.E.S. 2013. A multiple feature vector framework for forest species recognition. New York, New York, USA, Proc. of the ACM Symposium on Applied Computing, pp. 16 20.

Hafemann, L.G., Oliveira, L.S., Cavalin, P. 2014. Forest Species Recognition Using Deep
Convolutional Neural Networks. 22nd International Conference on Pattern Recognition (ICPR), pp. 1103 1107 .

Haindl, M., Vácha, P. 2015. Wood Veneer Species Recognition Using Markovian Textural Features. In: Computer Analysis of Images and Patterns, Lecture Notes in Computer Science. Springer International Publishing, Cham, pp. 300 311.

He, K., Zhang, X., Ren, S., Sun, J. 2016. Deep Residual Learning for Image Recognition. 2016 IEEE Conference on Computer Vision and Pattern Recognition (CVPR), pp. 770 778.

Hiremath, P.S., Bhusnurmath, R.A. 2017. Multiresolution LDBP descriptors for texture classification using anisotropic diffusion with an application to wood texture analysis. Pattern Recognition Letters 89: 8 17.

Hu, S., Li, K., Bao, X. 2015. Wood species recognition based on SIFT keypoint histogram. 8th International Congress on Image and Signal Processing (CISP), pp. 702 706.

Huang, S., Cai, C., Zhao, F., He, D., Zhang, Y. 2009. An efficient wood image retrieval using SURF descriptor. 2009 International Conference on Test and Measurement (ICTM), pp. 55 58.

Kapp, M.N., Bloot, R., Cavalin, P.R., Oliveira, L.E.S. 2014. Automatic forest species recognition based on multiple feature sets. Proc. of the International Joint Conference on Neural Networks, pp. 1296 1303.

Kobayashi, K., Akada, M., Torigoe, T., Imazu, S., Sugiyama, J. 2015. Automated recognition of wood used in traditional Japanese sculptures by texture analysis of their low-resolution computed tomography data. Journal of Wood Science 61(6): $630 \sim 640$.

Kobayashi, K., Hwang, S.W., Lee, W.H., Sugiyama, J. 2017. Texture Analysis of Stereograms of Diffuse-Porous Hardwood: Identification of 
Wood Species Used in Tripitaka Koreana. Journal of Wood Science 63: 322 330.

Khairuddin, A.S.M., Khalid, M., Yusof, R. 2011. Using Two Stage Classification for Improved Tropical Wood Species Recognition System. In: Intelligent Interactive Multimedia Systems and Services, Smart Innovation, Systems and Technologies. Springer Berlin Heidelberg, Berlin, Heidelberg, pp. 305 314.

Khalid, M., Yusof, R., Khairuddin, A.S.M. 2011. Tropical wood species recognition system based on multi-feature extractors and classifiers. Proc. of 2011 2nd International Conference on Instrumentation Control and Automation (ICA), pp. $6 \sim 11$.

Krizhevsky, A., Sutskever, I., Hinton, G.E. 2012. ImageNet classification with deep convolutional neural networks. Proc. of the 25th International Conference on Neural Information Proc. Systems, pp. 1097 1105.

Lecun, Y., Bottou, L., Bengio, Y., Haffner, P. 1998. Gradient-based learning applied to document recognition. Proceeding of the IEEE 86(11): $2278 \sim 2324$.

Martins, J.G., Oliveira, L.S., Britto, A.S., Sabourin, R. 2015. Forest species recognition based on dynamic classifier selection and dissimilarity feature vector representation. Machine Vision and Applications 26(2-3): 279 293.

Martins, J., Oliveira, L.S., Nisgoski, S., Sabourin, R. 2013. A database for automatic classification of forest species. Machine Vision and Applications 24(3): $567 \sim 578$.

Mohan, S., Venkatachalapathy, K., Sudhakar, P. 2014. An intelligent recognition system for identification of wood species. Journal of Computer Science 10(7): 1231 1237.

Nasirzadeh, M., Khazael, A.A., Bin Khalid, M. 2010. Woods recognition system based on local binary pattern. Proc. of 2nd International Conference on Computational Intelligence, Communication Systems and Networks (CICSyN 2010), pp. 308 $\sim 313$.

Paula Filho, P.L., Oliveira, L.S., Nisgoski, S., Britto, A.S.J. 2014. Forest species recognition using macroscopic images. Machine Vision and Applications 25(4): 1019 1031.

Peng, Z. 2013. Robust wood species recognition using variable color information. Optik International Journal for Light and Electron Optics 124(17): 2833 2836.

Simonyan, K., Zisserman, A. 2014. Very Deep Convolutional Networks for Large-Scale Image Recognition. arXiv.org. cs.CV.

Szegedy, C., Liu, W., Jia, Y., Sermanet, P., Reed, S., Anguelov, D., Erhan, D., Vanhoucke, V., Rabinovich, A. 2014. Going Deeper with Convolutions. arXiv:1409.4842.

Tou, J.Y., Tay, Y.H., Lau, P.Y. 2009a. A Comparative Study for Texture Classification Techniques on Wood Species Recognition Problem. Fifth International Conference on Natural Computation, pp. 8〜12.

Tou, J.Y., Tay, Y.H., Lau, P.Y. 2009b. Rotational invariant wood species recognition through wood species verification. 1st Asian Conference on Intelligent Information and Database Systems (ACIIDS 2009), pp. 115 120.

Wang, H.J., Zhang, G.Q., Qi, H.N. 2013a. Wood Recognition Using Image Texture Features Wu, R. (ed.). PLoS ONE 8(10): e76101 12 .

Wang, H.-J., Qi, H.-N., Wang, X.-F. 2013b. A new Gabor based approach for wood recognition. Neurocomputing 116: 192 200.

Yadav, A.R., Anand, R.S., Dewal, M.L., Gupta, S. 2014. Analysis and classification of hardwood species based on Coiflet DWT feature extraction and WEKA workbench. 2014 International 
Ohkyung Kwon $\cdot$ Hyung Gu Lee $\cdot$ Mi-Rim Lee $\cdot$ Sujin Jang $\cdot$ Sang-Yun Yang $\cdot$ Se-Yeong Park $\cdot$ In-Gyu Choi $\cdot$ Hwanmyeong Yeo

Conference on Signal Proceeding and Integrated Netwroks (SPIN), pp. 9 13.

Yadav, A.R., Dewal, M.L., Anand, R.S., Gupta, S. 2013. Classification of hardwood species using ANN classifier. Fourth National Conference on Computer Vision, Pattern Recognition, Image Processing and Graphics (NCVPRIPG), pp. 1 5. Yu, H., Cao, J., Luo, W., Liu, Y. 2009. Image retrieval of wood species by color, texture, and spatial information. 2009 International Conference on Information and Automation (ICIA), pp. 1116 1119.

Yusof, R., Rosli, N.R. 2013. Tropical Wood Species Recognition System Based on Gabor Filter as Image Multiplier. 2013 International Conference on Signal-Image Technology \& Internet-Based Systems (SITIS), pp. $737 \sim 743$.

Yusof, R., Khalid, M., Khairuddin, A.S.M. 2013a. Application of kernel-genetic algorithm as non- linear feature selection in tropical wood species recognition system. Computers and Electronics in Agriculture 93: 68 77.

Yusof, R., Khalid, M., Mohd Khairuddin, A.S. 2013b. Fuzzy logic-based pre-classifier for tropical wood species recognition system. Machine Vision and Applications 24(8): 1589 1604.

Yusof, R., Rosli, N.R., Khalid, M. 2010. Using Gabor Filters as Image Multiplier for Tropical Wood Species Recognition System. 12th International Conference on Computer Modelling and Simulation, pp. 289 294.

Zamri, M.I.P., Khairuddin, A.S.M., Mokhtar, N., Yusof, R. 2016. Wood Species Recognition System based on Improved Basic Grey Level Aura Matrix as feature extractor. Journal of Robotics, Networking and Artificial Life 3(3): $140 \sim 143$. 